\title{
The need to pursue and publish clinical trials in nanomedicine
}

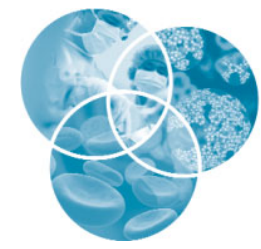

James R. Baker, Jr.

$\mathrm{W}$ hen I became Editor in Chief of Wiley's WIREs Nanomedicine and Nanobiotechnology, I felt one of the most important issues was that the publication is dedicated to nanomedicines. This essentially suggested that nanotechnology would be transitioning into the actual development of human therapeutics. Recently, this movement has begun. We have seen the initiation of multiple clinical trials of nanomaterials both for therapeutics and for medical devices. While this is exciting and encouraging, it is important to realize that nanotechnology now has to come to grips with the regulatory issues associated with a therapeutic approval process. This process is complex and expensive, but is necessary regardless of the nanomaterial or application. Many individuals who specialize in material science, analytical chemistry, or physics have not had prior experience with the regulatory process for either human therapeutics or medical devices. As such, I think it is worthwhile to review briefly the approval process for a human therapeutic.

First, while the procedures required to approve a drug were varied around the world, there has been recent convergence to these processes facilitated by the International Conference on Harmonization. Fundamental to the development process is the ability to characterize critical product attributes that ensure the identity, strength, quality, purity, and potency of the final product. New technologies will require new approaches to accomplish this assessment. In addition, at least four different sets of studies are necessary before a product can be submitted for approval for use in humans. Animal toxicity studies must first be done and under processes that are called good laboratory practices (GLP). These studies often require that more than one species be tested, usually a rodent and another non-rodent species, to ensure that anticipated and even unexpected toxicities are identified and characterized so the material can be

University of Michigan, Michigan Nanotechnology Institute for Medicine and Biological Sciences, Ann Arbor, Michigan, United States

DOI: 10.1002/wnan.141 given safely in the doses that are anticipated in human studies.

Animal studies are normally followed by three phases of human drug development. Phase I studies involve assessment of safety, tolerability, and pharmacokinetics aimed at determining maximum tolerated doses and certain pharmacokinetic parameters. Phase II studies are exploratory, dose ranging studies in carefully selected patients with the target indication of interest to further define safety and tolerability as well as demonstrate preliminary evidence of efficacy. Finally, Phase III studies are then initiated to confirm efficacy and safety in the patient population most likely to receive treatment post-approval. These large-scale studies are usually done in duplicate, and require an extensive number of subjects to provide adequate power to show that a clinical effect, if present, can be observed. The complex development and approval process for drugs therefore often requires up to 10 years, and hundreds of millions of dollars. In contrast, the approval process for medical devices often is shorter and requires fewer numbers of studies and subjects. Primarily, the device must be shown to function appropriately for its intended use without overt difficulties. However, because of recent problems with medical devices, this process has been made more rigorous. In some cases, it may now require a similar series of studies to the approval process for therapeutics.

As a result, although a research scientist often thinks that a drug is 'finished' when research ends and clinical development begins, it is just the start of a long and arduous process to obtain regulatory approval. It is clearly very important to understand exactly how nanomaterials can be developed into approved medicines and devices. As part of that process, we at WIREs Nanomedicine and Nanobiotechnology feel that the publication of preclinical and clinical studies related to therapeutic approval process is an important part of our job. Therefore, we particularly encourage authors to submit studies on therapeutic development of nanomedicines to our publication. We feel that there could be multiple aspects of these studies that would 
make excellent articles for WIREs Nanomedicine and Nanobiotechnology.

One area of interest would be an overview on the approach to obtaining regulatory approval for a new therapeutic. Studies that outline how specific therapeutics are to be tested and evaluated, both in terms of toxicity and clinical efficacy, would be important. Publishing this work would enhance our understanding of the process of nanotherapeutic and device approval as well as to get feedback from readers as to potential improvements in this process. Improving the efficiency of clinical trials is one important way to decrease the cost and time involved in therapeutic development. If we can help facilitate this goal through the publication of preliminary proposals, we believe that it would be an important goal for nanomedicine.

The second and most exciting area would be the publication of the results of clinical trials with nanomedicines. We encourage submission of the results of clinical trials that have both positive and negative findings. The positive trials will tell us about new therapeutics that are in development and present their unique activities for us to understand. Negative clinical trials may provide important lessons into problems associated with the development of nanomedicines or the use of nanomaterials in clinical trials. These 'lessons learned' may markedly improve the ability of other individuals to develop therapeutics. Also, publishing summaries of regulatory interactions related to development of nanomedicines would provide valuable insight into how nanotechnology is perceived within the regulated environment. It would be important to understand why a drug or a device has taken a specific path toward the clinic and what type of results will be or were necessary to achieve the approval. A final type of article we feel would be interesting to publish would be the personal perspectives of those involved in obtaining drug approval. Understanding the frustrations and challenges that are inherent in this process should certainly help others who are attempting to move products through development.

I hope you share my enthusiasm for the clinical development of nanomedicines. Those involved in this process have my utmost respect and I hope that they will share their work with us. If nothing else, having an open discussion about the hurdles in characterization of nanomedicines, assessment of their safety in animals and entry into clinical development will enhance the number of products that will become nanomedicines from nanomaterials. 